\title{
STUDY ON THE ADSORPTION PHENOMENON IN SHALE WITH THE COMBINATION OF MOLECULAR DYNAMIC SIMULATION AND FRACTAL ANALYSIS
}

\author{
LIEHUI ZHANG,${ }^{*, \boldsymbol{\Phi}, * *}$ JIANCHAO LI, ${ }^{*}$ DU JIA,${ }^{\dagger}$ YULONG ZHAO $,{ }^{*}, \|, * *$ CHUNYU XIE ${ }^{\ddagger}$ \\ and ZHENGWU TAO $\$$ \\ *State Key Laboratory of Oil and Gas Reservoir Geology \\ and Exploitation Southwest Petroleum University \\ Chengdu, Sichuan 610500, P. R. China \\ ${ }^{\dagger}$ Research Center of China United Coalbed Methane Corporation, Ltd. \\ Beijing 100011, P. R. China \\ $\ddagger$ Environmental Safety and Technical Supervision Institute \\ Southwest Oil $\&$ Gas Field Company, PetroChina \\ Chengdu, Sichuan 610041, P. R. China \\ ${ }^{\S}$ Research Institute of Exploration and Development \\ Tarim Oilfield Company, PetroChina, Korla \\ Xinjiang 841000, P. R. China \\ \$zhangliehui@vip.163.com \\ 11373104686@qq.com
}

Received December 21, 2017

Accepted January 13, 2018

Published March 1, 2018

\begin{abstract}
As one of the key status of gas in shale reservoir, adsorption gas accounts for considerable percentage of total gas amount. Due to the complexity and nanostructure of shale gas reservoir, it is very challenging to represent adsorption gas through traditional methods. However, the integration of the fractal theory and molecular dynamics (MD) simulation may provide a new perspective of understanding such nanostructure and the micro-phenomenon happening in it.
\end{abstract}

\footnotetext{
** Corresponding authors.

This is an Open Access article published by World Scientific Publishing Company. It is distributed under the terms of the Creative Commons Attribution 4.0 (CC-BY) License. Further distribution of this work is permitted, provided the original work is properly cited.
} 
The key purpose of this paper is to investigate the adsorption phenomenon in shale kerogen. By using MD simulation and grand canonical Monte Carlo (GCMC) algorithm, the adsorption of methane in 2, 5 and $10 \mathrm{~nm}$ slit-like pores is simulated for different temperature and pressure status. According to the results, the average gas density in smaller pores is higher than that in bigger pores, and multilayer adsorption presents on some areas of pore surfaces. Then, the simulation results are analyzed using the multilayer fractal adsorption model. The analysis indicates that the number of adsorption layer increases with pressure increase: four-layer adsorption presents in $10 \mathrm{~nm}$ pores while three-layer adsorption shows up in $2 \mathrm{~nm}$ and $5 \mathrm{~nm}$ pores due to pore volume limit. Fractal dimension of pore wall surface generated in this study is in the range of 2.31-2.63. Moreover, high temperature could decrease the adsorption behavior in reservoir condition.

Keywords: Shale; Molecular Dynamics Simulation; Fractal; Adsorption.

\section{INTRODUCTION}

With the technology development, shale gas has become the important component of oil and gas resources. The methane in shale reservoir mainly exists in three status: free gas in pores and fractures, solution gas in kerogen matrix, and adsorption gas on the surfaces of kerogen and minerals. At the early stage of shale gas development, free gas dominates the production; while the adsorption gas which accounts for $20-85 \%$ of original gas in place (OGIP) 11 determines gas production in the later stage due to slow desorption mechanism in nanopores.2] Therefore, the adsorption status of methane in shale matrix is very important. Many literatures have studied the nanopore structure and adsorption behavior in shale, ${ }^{\sqrt[3]{6}}$ and the worldwide lab tests provide us tremendous data to investigate the adsorption phenomenon in different shale samples, ${ }^{7-14}$ however, these adsorption behaviors are yet to be explained theoretically.

Aiming at the unconventional characteristics of shale reservoir, cross-disciplinary theories and technologies have been applied and resulted in some meaningful achievements. Molecular dynamics (MD) simulation plays an important role in investigating phenomena in complex systems, and grand canonical Monte Carlo (GCMC) algorithm is a simulation method to analyze the adsorption phenomenon in micro-porous media. In this paper, the integration of MD simulation and GCMC technique is applied to analyze the adsorption behavior of methane in shale reservoir. Later on, a multilayer fractal adsorption model is used to match the isothermal adsorption data.

Many studies on methane adsorption in shale are based on simplified matrix assumptions. 15
However, shale kerogen system is much more complex in reality. In the study of Zhang et al. 18 actual components were used to construct coal model; and Sawa et al. ${ }^{[19}$ also used real kerogen molecule to represent shale matrix for adsorption study and received reasonable results. Therefore, in this paper, MD simulation is firstly applied to construct kerogen matrix; and then 2, 5 and $10 \mathrm{~nm}$ wide pores are generated in the randomly constructed matrix under different temperature and pressure status; thereafter, gas distribution in the slit-like pores is also generated by MD simulation; last, multilayer fractal adsorption model is used instead of Langmuir model to analyze adsorption parameters in such system in consideration of the presence of multilayer adsorption in nanopores.

\section{METHODOLOGY}

\subsection{Simulation}

In this paper, the construction of kerogen molecules is based on the study of Collell et al! $!^{20}$ and Vandenbroucke 21 : elements of C, H, O, N, S, etc. constitute kerogen molecules; and element ratios of $\mathrm{H} / \mathrm{C} \sim 0.5$, $\mathrm{O} / \mathrm{C} \sim 0.02, \mathrm{~S} / \mathrm{C} \sim 0.005$ and $\mathrm{N} / \mathrm{C} \sim 0.005$ are satisfied. These results came from a series of lab tests, including molecular weight, elemental composition and functional group spectrum analysis. This type of kerogen consists of $4-5$ polyaromatic clusters bridged by short alkyl chains. Figure 1 shows the typical fragment of this kind of kerogen molecule.

To construct the solid matrix of kerogen, multiple kerogen molecules are randomly generated based on the elemental ratios mentioned above and then relaxed by MD simulation. The kerogen solid in 


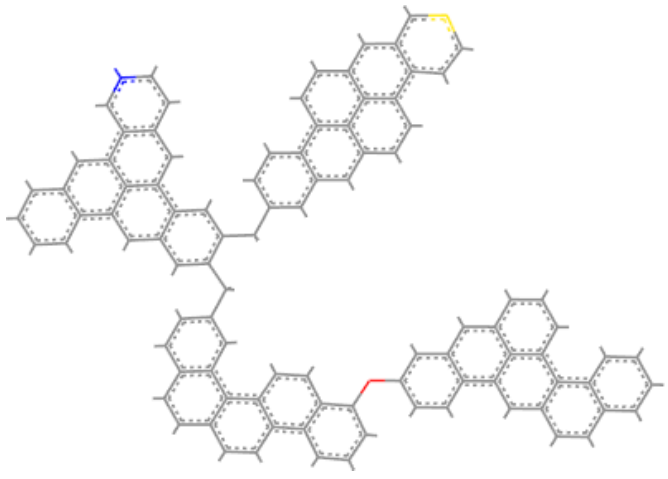

(a)

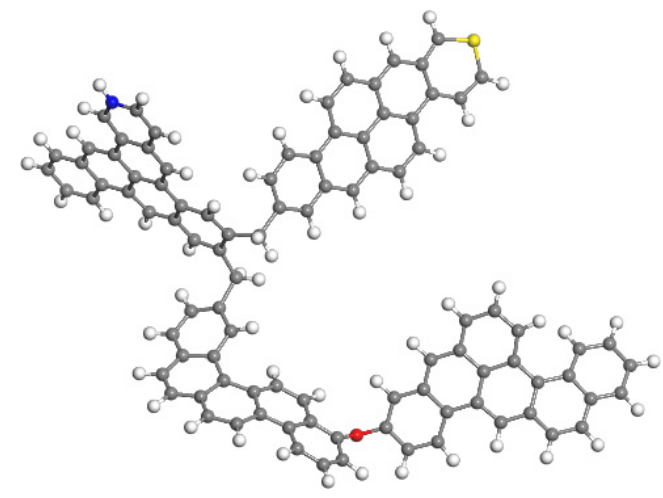

(b)

Fig. 1 (a) Chemical structure of kerogen molecular fragment and (b) geometry structure of kerogen fragment relaxed by MD simulation in which $\mathrm{C}, \mathrm{H}, \mathrm{O}, \mathrm{N}$ and $\mathrm{S}$ are indicated by clay, white, red, blue and yellow sphere.

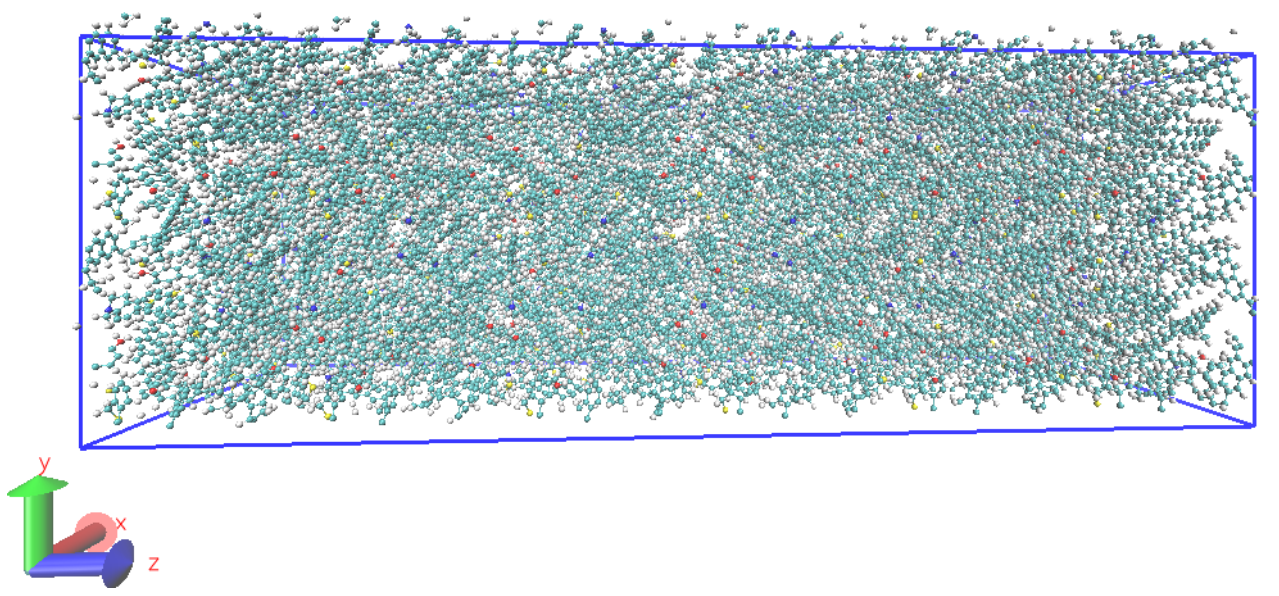

Fig. 2 Kerogen solid generated by MD simulation.

this study is constituted of more than 100 kerogen molecules, and simulated in an NPT ensemble with constant molecular number $N$, pressure $P$ and temperature $T$ by Lammps ${ }^{22}$ simulation under periodic boundary conditions. The force fields of inter- and intra-molecules are calculated by CVFF ${ }^{23}$ Meanwhile, according to the study of Gasparik et al.24 the density of kerogen simulated by MD varies very slightly within the temperature of $308-368 \mathrm{~K}$ and pressure of 5-20 Mpa, therefore the kerogen simulated under $20 \mathrm{Mpa}$ and $363 \mathrm{~K}$ is used for this analysis. In the end, the density of the simulated kerogen is $1.23 \mathrm{~g} / \mathrm{cm}^{3}$, and the dimension of the original simulation box is $5 \times 5 \times 14 \mathrm{~nm}^{3}$; the visualization of the simulation system is realized by visual MD (VMD) 1.9.1. ${ }^{25}$ Figure 2 shows the final kerogen state in the simulation box.

In this study, the slit-like pores which are most popular in kerogen solid are generated to represent gas storage space. The pore generation is through deleting molecules in the specified regions, including atom deletion and bond deletion. The sizes of pores are determined by the width of the deleted regions. Figure 3 shows a slit-like pore of $2 \mathrm{~nm}$ width generated by this method. Concentration profile of methane will be discussed in the pore volume space (Fig. 3b).

Because of its simple symmetrical structure, methane is treated as united atom of LennardJones (LJ)-type. Compared to full atom model, the united model has higher efficiency in calculation and therefore more appropriate to represent methane. For methane molecules, non-bonded Van der Waals force is accounted between atom particles, and for LJ-type force field, the LJ potential function can be expressed as

$$
E_{i j}=4 \varepsilon_{i j}\left[\left(\frac{\delta_{i j}}{r_{i j}}\right)^{12}-\left(\frac{\delta_{i j}}{r_{i j}}\right)^{6}\right], \quad r_{i j} \leq r_{\mathrm{cut}},
$$

where $\delta_{i j}$ is the distance between the two particles when the potential energy is zero; $\varepsilon_{i j}$ is the depth of 


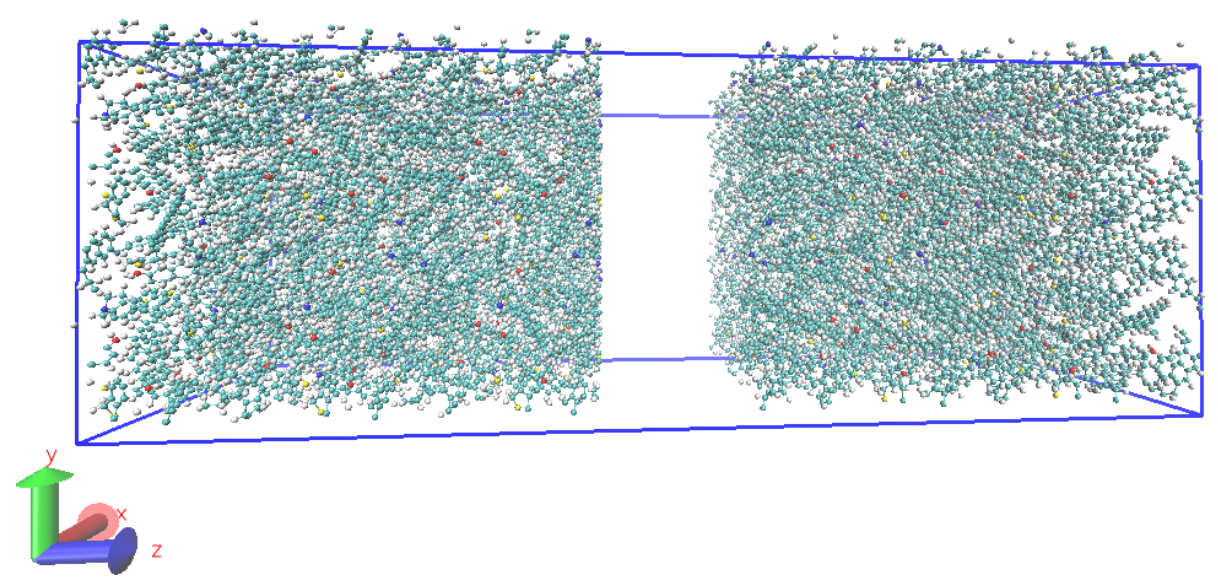

(a)

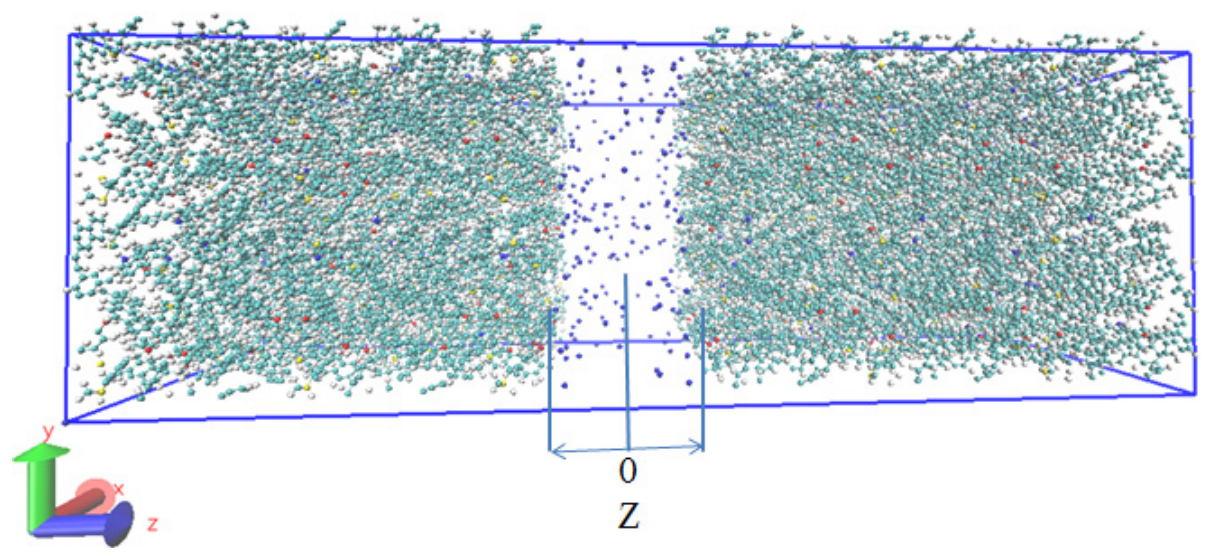

(b)

Fig. 3 (a) Slit-like pore in the kerogen solid and (b) the state of pore filled with methane gas.

the potential well; $r_{i j}$ is the distance between atom $i$ and atom $j$. Interaction longer than $r_{\text {cut }}$ is omitted, since the weak interaction beyond this distance is negligible.

The interactions between kerogen and methane molecules are calculated by Lorentz-Berthelo mixing rule:

$$
\begin{aligned}
\delta_{i j} & =\frac{1}{2}\left(\delta_{i}+\delta_{j}\right), \\
\varepsilon_{i j} & =\sqrt{\varepsilon_{i} \varepsilon_{j}} .
\end{aligned}
$$

Relevant parameters of the LJ potential can be referred in Refs. 26 and 27. In this study, a cutoff radius of $9.5 \AA$ is used in LJ calculation. Table 1 gives the LJ potential parameters of methane used in this simulation.

\subsection{GCMC Simulation}

The GCMC algorithm can perform exchanges for given types of atoms or molecules in ideal gas reservoir at the specified temperature $T$ and
Table 1 LJ Potential Parameters of Methane Used in the Current Simulation.

\begin{tabular}{crr}
\hline & $\varepsilon / k_{B}(\mathbf{K})$ & $\delta(\AA)$ \\
\hline $\mathrm{CH}_{4}$ & 148.1 & 3.81 \\
$\mathrm{C}$ & 28.1 & 3.40 \\
\hline
\end{tabular}

chemical potential $\mu \cdot \cdot^{28129}$ In the grand canonical ensemble $(\mu V T$, constant chemical potential, constant volume, and constant temperature), isothermal adsorptions of micro-porous media can be simulated and the chemical potential of given status can be solved as

$$
\mu=k_{B} T \ln \frac{\phi P \Lambda^{3}}{k_{B} T},
$$

where $\Lambda$ is the thermal de Broglie wavelength; $k_{B}$ is Boltzman's constant; $\phi$ is the fugacity coefficient, which can be calculated by $P R$ equation of state. 
Thus, the chemical potential is related to pressure, temperature and the gas property.

The GCMC simulation attempts to insert or delete gas atoms with specific time steps. The acceptance probability formula can be found in Ref. [30. When the chemical potential and temperature between the adsorbed phase and gas phase reach an equivalence, the adsorption process also comes to a balance. The atom coordinates and other relevant properties can be acquired from the statistical data.

The adsorption parameter resulted from the experiment is excess adsorption $n_{\mathrm{ex}}\left(\mathrm{cm}^{3} / \mathrm{g}\right)$, which is defined as the extra amount of adsorption gas addition to the equivalent volume of the bulk phase gas in unit pore volume. Since the calculation in the traditional isothermal adsorption model is based on the absolute adsorption $n_{\mathrm{ab}}\left(\mathrm{cm}^{3} / \mathrm{g}\right)$, the conversion between these two parameters can be expressed as $31+36$

$$
n_{\mathrm{ab}}=\frac{n_{\mathrm{ex}}}{1-\frac{\rho_{g}}{\rho_{\text {adsorbed }}}},
$$

where $\rho_{g}$ is the density of gas $\left(\mathrm{g} / \mathrm{cm}^{3}\right) ; \rho_{\text {adsorbed }}$ is the density of adsorbed gas $\left(\mathrm{g} / \mathrm{cm}^{3}\right)$.

Through the above equation, the excess adsorption gas is converted into the absolute value. However, the assumption of treating adsorption gas as liquid state is questionable. In MD simulation, such problem can be solved by calculating the adsorption gas density directly, hence, the density parameter $\rho_{\text {adsorbed }}$ in Eq. (5) is modified and calculated directly from the molecular simulation.

\subsection{Fractal Analysis in Shale Reservoir}

Adsorption behavior in shale is widely fitted by Langmuir model, however, Langmuir adsorption model assumes monolayer adsorption and there is no interaction between adsorbed molecules, which makes it questionable when multilayer adsorption phenomenon is found. The adsorption potential model like $\mathrm{D}-\mathrm{A}$ model and $\mathrm{D}-\mathrm{R}$ model can overcome the deficiency of Langmuir model and forecast the multilayer adsorption behavior, but they cannot clearly describe the specific adsorption state, the adsorption number calculated by these models is the total adsorption number, the multilayer behavior is still unsolved. So, a model can both be used in the multilayer adsorption and can describe the adsorption behavior clearly which is needed.
Fractal geometry is widely used in complex systems to describe the behavior and phenomenon happened in it!37,42 Plenty of studies find fractal characters in shale reservoir, the fractal pore size distribution in shale greatly influences the gas storage, and the fractal surface of pore wall has huge impact on gas distribution in pore. The current study focuses on the adsorption behavior of methane on pore walls, so we will discuss the influence of fractal surface of pore wall on methane adsorption.

For molecules adsorbed on specific surface area, the number of molecules is decided by its profile radius $r$. For monolayer adsorption, a surface with fractal dimension $D$, the relationship between saturate adsorption number $n_{m}$ and radius $r$ is

$$
n_{m} \propto r^{-D} \text {. }
$$

For multilayer adsorption, the relationship between the first layer adsorption number $N_{1}$ and the $i$ th layer adsorption number $N_{i}$ is 43

$$
f_{i}=\frac{N_{i}}{N_{1}}=i^{-(D-2)} .
$$

In our previous study, 44 the multi-layer fractal adsorption model (F-BET) is proposed to describe the adsorption in nanostructure at supercritical condition, and the adsorption data in the previous work come from isothermal adsorption experiment. In this study, the results from the MD simulation will be analyzed by the multi-layer fractal adsorption model, which is written as

$$
V=\frac{V_{m} C \sum_{i=1}^{n} i^{2-D_{s}} \sum_{j=i}^{n} x^{j}}{1+C \sum_{i=1}^{n} x^{i}},
$$

where $x$ represents the relative pressure $\frac{P}{P_{0}}$;

$$
C=\frac{a_{1} b_{n}}{b_{1} a_{n}} \exp \left(\frac{E_{1}-E_{L}}{R T}\right),
$$

where adsorption constant $a_{1}$ and desorption constant $b_{1}$ are fixed values; $R$ is the gas constant; $n$ is number of adsorption layer; $V_{m}$ is the monolayer saturation adsorption volume, adsorption heat of the first layer $\left(E_{1}\right)$ is a fixed value, the following layers' adsorption heat is the heat of liquefaction $\left(E_{L}\right) . D_{s}$ is the fractal dimension of adsorption surface 45 :

$$
D_{s}=\lim _{r \rightarrow 0} \frac{\ln S_{r}}{\ln r} .
$$

Here, $S_{r}$ is the surface of the adsorbent measured on a length scale $r$. If $D_{s}=2$, it becomes the BET 
model:

$$
\frac{V}{V_{m}}=\frac{C x\left[1-(n+1) x^{n}+n x^{n+1}\right]}{(1-x)\left[1+(C-1) x-C x^{n+1}\right]} .
$$

When $n \rightarrow \infty$, Eq. (8) is simplified as

$$
V=\frac{V_{m} C}{1-x-C x} L i_{D_{s}-2}(x)
$$

or

$$
V=\frac{V_{m}}{1-x-C x} \sum_{i=1}^{\infty} \frac{x^{i}}{i^{D_{s}-2}} .
$$

Here, the adsorption parameters are calculated first by the BET model. In this model, the pore surfaces are assumed flat with fractal dimension of 2 . Then, the multilayer adsorption behavior is matched by the F-BET model, in which the number of the adsorption layers is based on the results from the BET model. Also, when supercritical conditions occur in the simulation process, the pseudosaturation vapor pressure is calculated by Dubinin's method ${ }^{46}$ as follows:

$$
P_{s}=P_{c}\left(\frac{T}{T_{c}}\right)^{2}
$$

where subscript $c$ represents critical point, for example, critical pressure $P_{c}$ of methane is $4.5992 \mathrm{MPa}$ and critical temperature $T_{c}$ is $190.56 \mathrm{~K}$.

\section{RESULTS AND DISCUSSION}

\subsection{Simulation Details}

To get the adsorption information of methane in the kerogen solid system, the adsorption processes are simulated by Lammps simulator assuming different temperature and pressure conditions. As described in Sec. 2.1, the slit-like pores of 2, 5 and $10 \mathrm{~nm}$ width are constructed, respectively, and the simulation box size is $5 \times 5 \times 14 \mathrm{~nm}^{3}$.

The GCMC insertion and deletion is exerted at different temperatures: (333 K, $363 \mathrm{~K})$ and within pressure range of $1-25 \mathrm{Mpa}$. The parallel simulation is run on a 48-core Dell workstation with time step of $1 \mathrm{fs}$ and $10^{7}$ steps for each simulation.

\subsection{Adsorbed Methane Density}

Figures 46 show the distributions of methane in kerogen pores of different width under specific temperature $(333 \mathrm{~K})$ and pressure, which clearly reflects the status of methane under various conditions. $Z$ variable means the distance to the pore center in $Z$-axis direction, $\mathrm{CH}_{4}$ number means the total number of methane on the $x y$ plane with corresponding $Z$ value (see Fig. 3 b). It can be seen that, under given temperature and pressure conditions, pore structure greatly influences gas distribution:

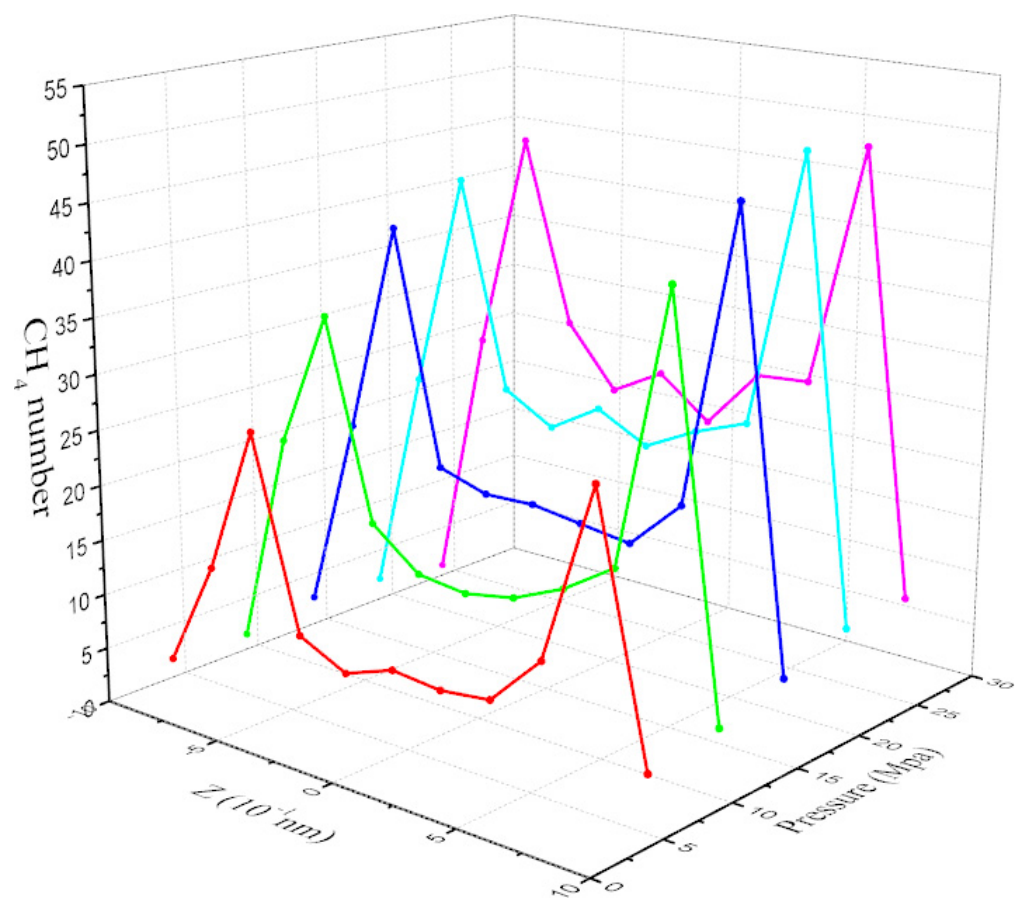

Fig. 4 Methane number distribution in $2 \mathrm{~nm}$ kerogen pore at $333 \mathrm{~K}$. 


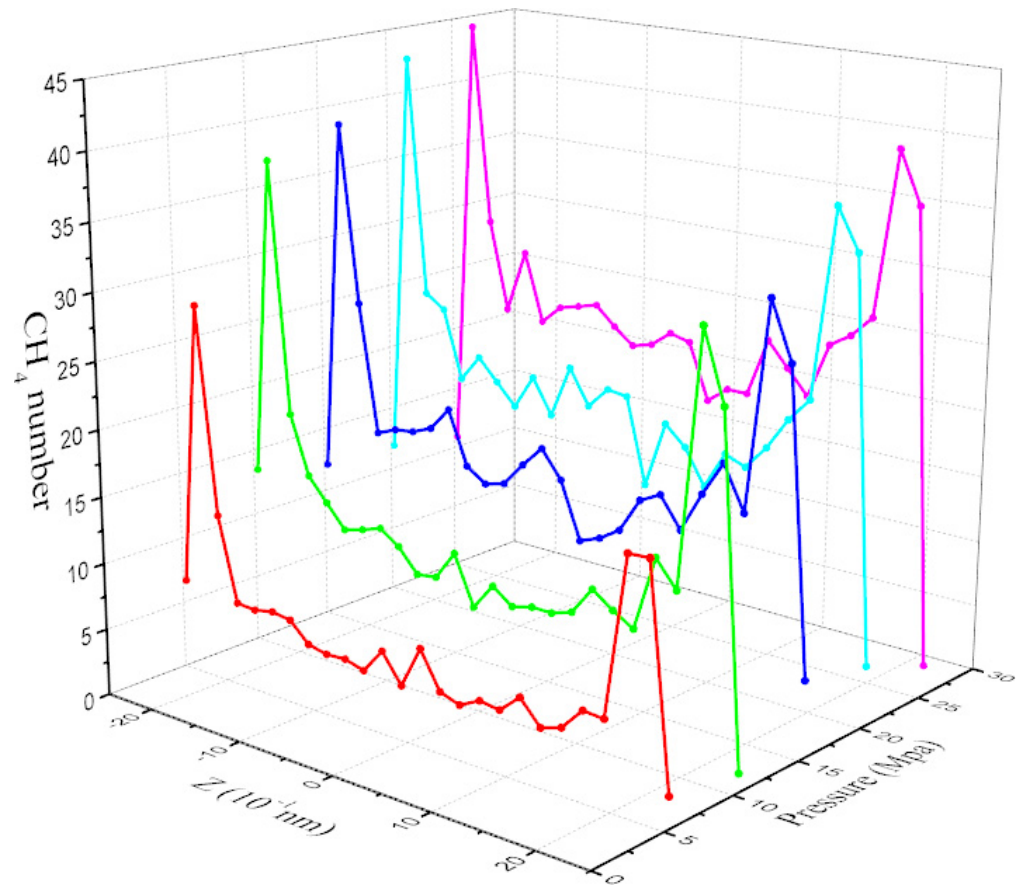

Fig. 5 Methane number distribution in $5 \mathrm{~nm}$ kerogen pore at $333 \mathrm{~K}$.

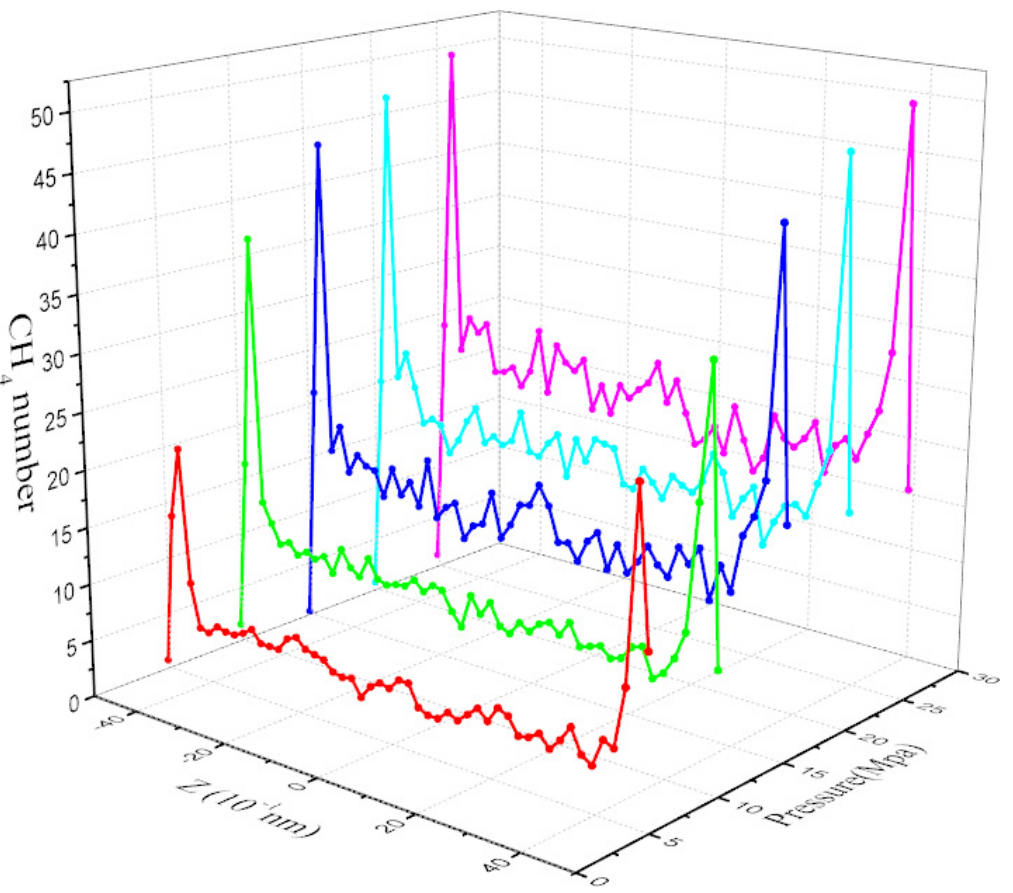

Fig. 6 Methane number distribution in $10 \mathrm{~nm}$ kerogen pore at $333 \mathrm{~K}$.

average gas density is higher in smaller pores; the adsorption layers on the surfaces of big pores diminish with increasing distance to pore surfaces. This phenomenon is highly because of the interaction forces between pore surfaces of kerogen media. In small pores $(2 \mathrm{~nm})$, the pore surfaces are so close and even within the cutoff radius of pair interaction, thus, the methane molecules have interactions with both sides of pore surfaces, which leads to comparatively even gas distribution; while in big pores $(10 \mathrm{~nm})$, the surface distance is far bigger than the cutoff radius of pair interaction, and therefore, the gas molecules along the pore surfaces only react with one surface side and those in the middle of 


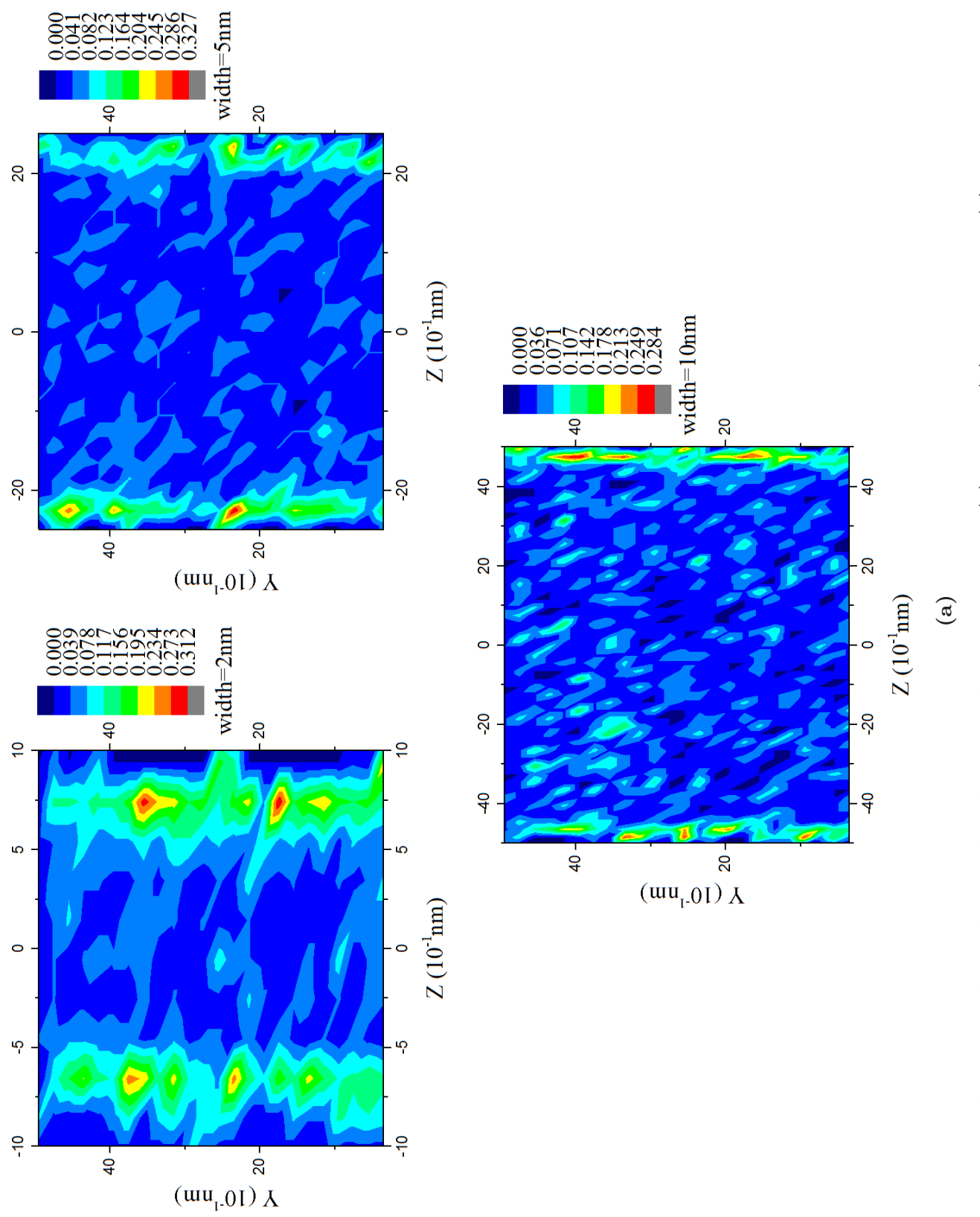




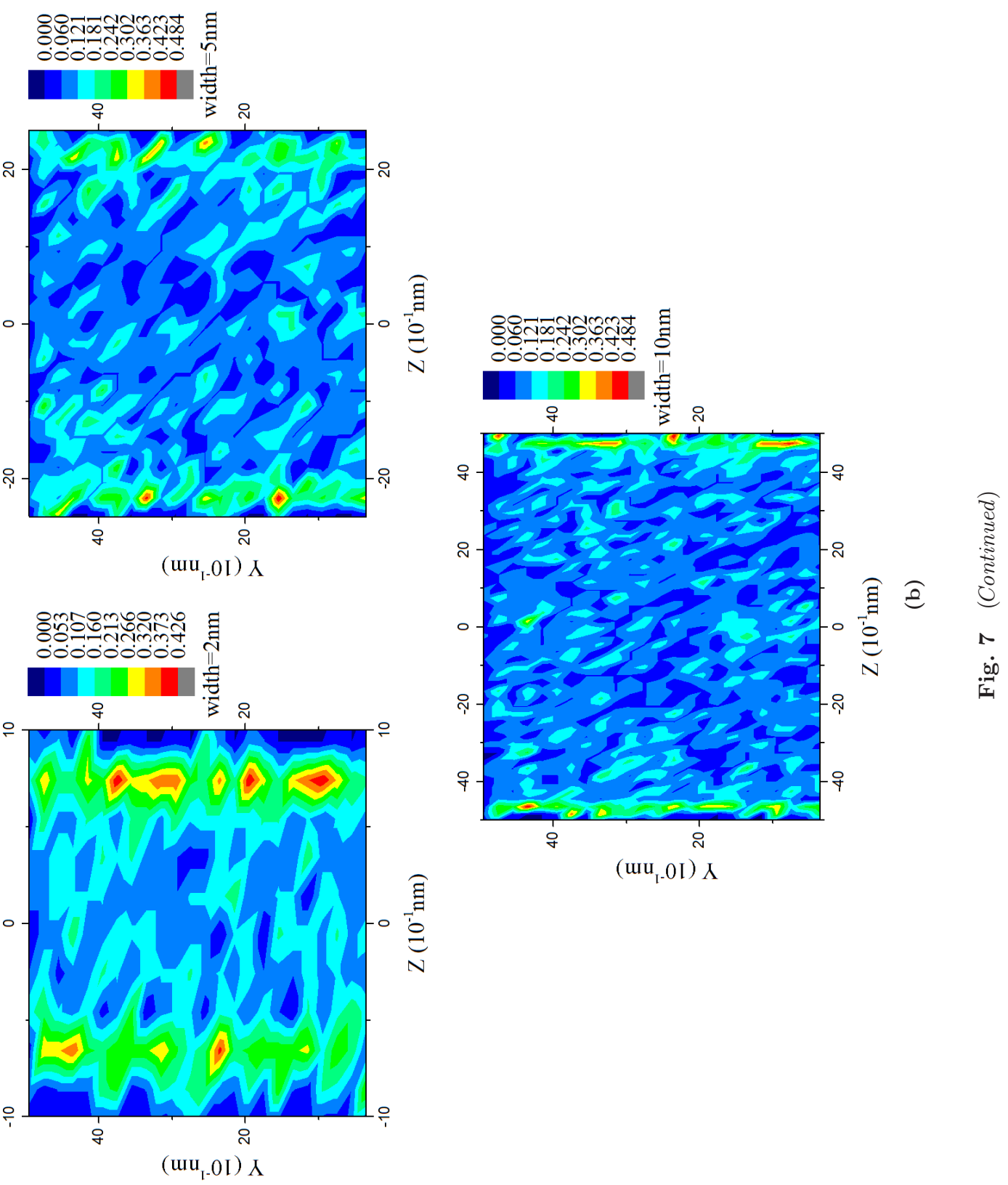


L. Zhang et al.

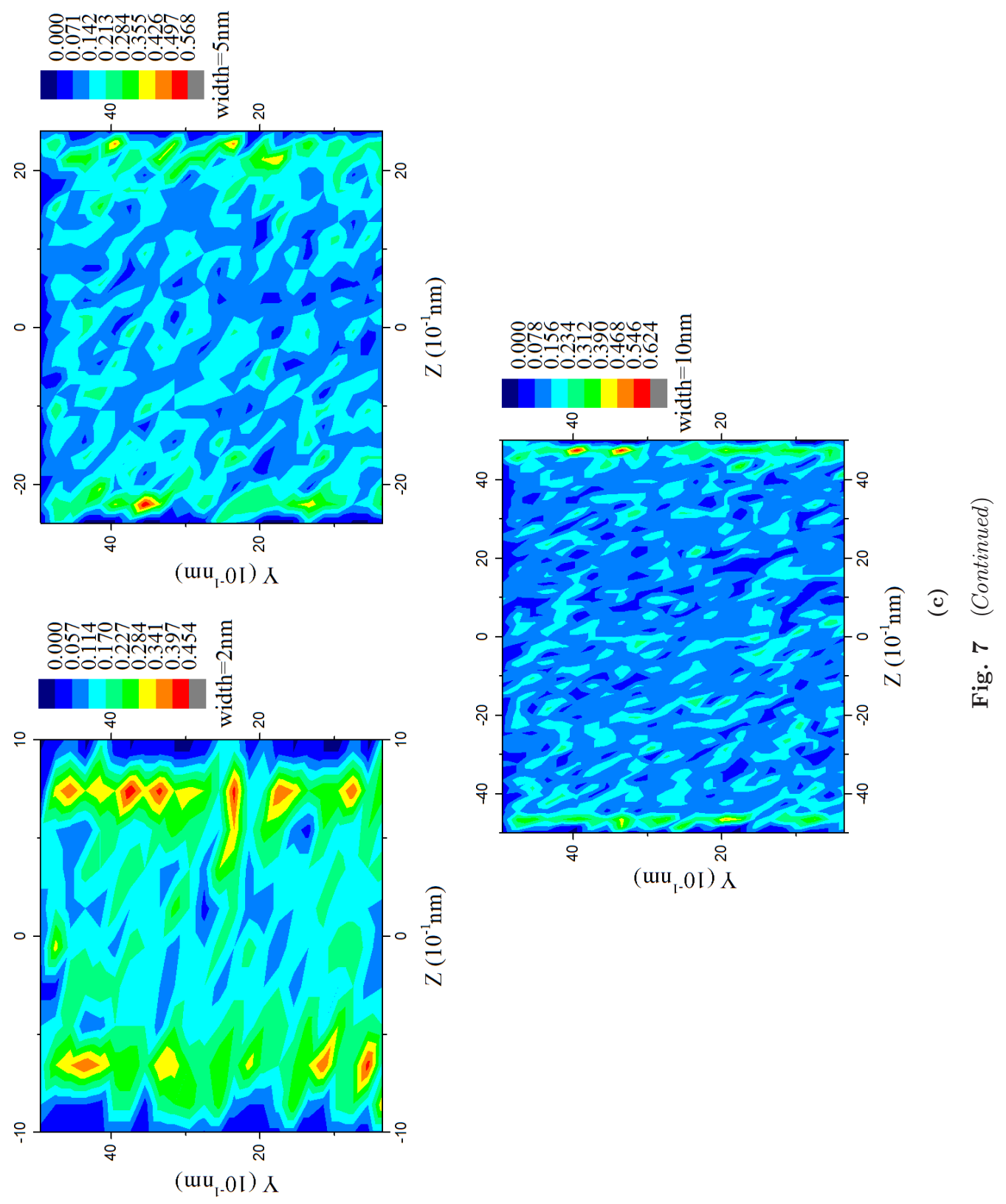


pores only react with each other. From these results, it is observed that methane adsorption in nm kerogen pores is multilayer adsorption, which is more clear in Fig. 7.

Figure 7 shows the profile of methane density in kerogen pores under given temperature and pressure conditions. $Z$ variables represent the distance to the center of the pore in $Z$-axis direction, the figure represents the density distribution of methane on the $y z$ plane and the density number is the accumulation of methane molecules of all the $X$-axis value on $y z$ plane (see Fig. 3b).

The free gas and adsorption gas can be differentiated easily. Controlled by pore volume, pore filling phenomenon is very obvious in the $2 \mathrm{~nm}$ wide pore: the average gas density is higher than that in other two pore sizes even under $5 \mathrm{MPa}$ pressure; and adsorption layers accumulate quickly along pore surfaces. Similarly, due to weak interaction between pore surfaces and methane molecules, the average gas density is the lowest in the $10 \mathrm{~nm}$ pore, and the adsorption layers are also thinner than those in the $2 \mathrm{~nm}$ pore. With system pressure increases, the gas status changes dramatically: the boundaries of adsorption gas and free gas become blurred in small pores while they are still clear in bigger pores. Meanwhile, it is also noted that the adsorption layers increase with increase in pressure. The gas density profiles of different sizes of pores indicate that small pores have higher gas storage capacity than bigger pores of the same pore volume.

Another observation from this analysis is that the distribution of methane molecules on pore surfaces is not even. This can be explained in two reasons: one is the components of kerogen, the different interactions between different kerogen atoms and methane molecules lead to such uneven distribution, which is one of the effects by simulating real kerogen molecules; another is the roughness of kerogen pores, which makes it more important to describe pore surface roughness in the adsorption models.

The isothermal adsorption curves can be plotted by adsorption gas and free gas density data: in this study, the excess adsorption volume is replaced by the absolute adsorption volume, which can be acquired by calculating average density profile of free gas in pores. It can be seen in the density profile that there are boundaries between adsorption layers and free gas. The absolute adsorption volume can be calculated through adding up the gas amount of all adsorption regions.

\subsection{Fractal Analysis of Methane Adsorption in Kerogen}

This paper focuses on the adsorption behavior of methane in slit-like pores of kerogen. In previous sections, the parameters of methane adsorption in kerogen pores of different sizes are acquired for different temperature and pressure conditions. In this section, a multilayer fractal adsorption model will be used to match and acquire isothermal adsorption data. Such multilayer model is more appropriate than Langmuir single layer model according to what has been observed in this study.

The adsorption layer can be identified from the gas density profile: under given temperature and pressure conditions, gas density is a constant and the density of the adsorption layers is higher than free gas, which is used to justify the two gas status. As shown in Figs. 4 and [7, it is easy to differentiate adsorption layers and free gas.

The first step is to match previous simulation results from the MD model by the BET model. Table2 lists the results under temperature $333 \mathrm{~K}$. In the range of simulation pressure $(0-25 \mathrm{Mpa})$, a flat pore surface with fractal dimension of 2 can have more layers in bigger pores, this is because of the pore volume limitation of smaller pores.

The isothermal adsorption curves for different pore width are shown in Fig. 8 The plot data are acquired by the MD simulation while the curves are generated through the BET match. As shown in the plot, the adsorption capacity of pores increases with pore width. However, due to pressure limitation and similarity of pore surface properties, the adsorption capacity of different pores varies only slightly. According to these curves, it can be predicted that the adsorption volume of big pores and small pores can vary great enough once the pressure increases to certain level, where adsorption continues in bigger pores while ceases in smaller pores due to pore volume limitation. Similarly, the isothermal adsorption curves at temperature $363 \mathrm{~K}$ are generated. It can

Table 2 BET Model Simulation Results at $333 \mathrm{~K}$.

\begin{tabular}{cccccc}
\hline $\begin{array}{c}\text { Pore Width } \\
(\mathbf{n m})\end{array}$ & $\begin{array}{c}\boldsymbol{V}_{\boldsymbol{m}} \\
\left(\mathbf{C H}_{4} \text { number }\right)\end{array}$ & $\boldsymbol{C}$ & $\boldsymbol{n}$ & $\boldsymbol{R}^{\mathbf{2}}$ & $\boldsymbol{D}_{\boldsymbol{s}}$ \\
\hline 2 & 103.95 & 3.59 & 2.3 & 0.998 & 2 \\
5 & 112.76 & 3.26 & 2.2 & 0.998 & 2 \\
10 & 93.72 & 5.9 & 2.65 & 0.998 & 2 \\
\hline
\end{tabular}

Note: $R^{2}$ is the correlation coefficient, a bigger value close to 1 reflects a more accurate simulation. 
be seen that increasing temperature leads to reducing adsorption gas volume under a give pressure condition (reservoir pressure $25 \mathrm{MPa}$ ), it means in reservoir condition, higher temperature will reduce the adsorbed methane.

Based on the BET match, the multilayer fractal adsorption model is used to calculate adsorption parameters on rough pore surfaces, which have fractal dimension of 2-3. As mentioned in the previous study, ${ }^{32}$ the rough surfaces cause more adsorption layers. Therefore, the number of adsorption layers from the fractal multilayer model will increase on the basis of layers from the BET model.

Table 3 lists the results for pores of different sizes. In $2 \mathrm{~nm}$ and $5 \mathrm{~nm}$ pores, three layers reach the adsorption limit and the fourth layer resulted in fractal dimension greater than 3 , which is not allowable; in the $10 \mathrm{~nm}$ pore, more layers can be adsorbed, but maximize at 4 when the fractal dimension becomes 3.25 for the fifth layer, which is also not allowable. According to such results, bigger pores have higher adsorption capacity than smaller pores, which matches the prediction of the isothermal adsorption curves.

During the construction of slit-like pores in kerogen matrix, some kerogen molecules were deleted, which leads to rough pore surfaces. Therefore, the multilayer fractal adsorption model representing rough surfaces is more applicable here than the BET model representing smooth surfaces. As shown in Table 3, the same adsorption volume takes more layers with the increase in fractal dimension (more complex surface conditions), this means thicker adsorption area on rough surfaces than smooth
Table 3 F-BET Model Methane Adsorption Simulation Results at $333 \mathrm{~K}$.

\begin{tabular}{clclll}
\hline $\begin{array}{c}\text { Pore } \\
\text { Width (nm) }\end{array}$ & $\boldsymbol{n}$ & $\begin{array}{c}\boldsymbol{V}_{\boldsymbol{m}} \\
\left(\mathbf{C H}_{4} \text { number) }\right.\end{array}$ & $\boldsymbol{C}$ & $\boldsymbol{R}^{\mathbf{2}}$ & $\boldsymbol{D}_{\boldsymbol{s}}$ \\
\hline \multirow{2}{*}{2} & 2.3 & 103.95 & 3.59 & 0.998 & 2 \\
& 3 & 103.28 & 3.9 & 0.999 & 2.63 \\
& 4 & 104.78 & 4.13 & 0.998 & 3.07 \\
5 & 2.2 & 112.76 & 3.26 & 0.998 & 2 \\
& 3 & 107.23 & 3.82 & 0.996 & 2.62 \\
& 4 & 108.71 & 4.01 & 0.996 & 3.11 \\
10 & 2.65 & 93.72 & 5.9 & 0.998 & 2 \\
& 3 & 95.71 & 6.04 & 0.998 & 2.31 \\
& 4 & 102.38 & 5.86 & 0.997 & 2.93 \\
& 5 & 105.68 & 5.81 & 0.997 & 3.25 \\
& & & & & \\
\hline
\end{tabular}

surfaces, which causes pore space reduction for free gas.

What is more, we can find a trend in Table 3: the monolayer saturation adsorption number $V_{m}$ is increased with the increase of fractal dimension $D_{s}$, this is because larger $D_{s}$ means a more complicated wall surface, and this kind of surface will have bigger capacity than the flat surface.

The problem we confront is that we cannot determine the fractal dimension or the number of adsorption layer directly from the simulation, if one of them can be decided, then we can use F-BET model to calculate the other parameter. But, we still can estimate the two parameters based on Figs. 7 ; and 8 in Fig. 8, at $25 \mathrm{Mpa}$ and $333 \mathrm{~K}$, adsorbed number of methane in 2, 5 and $10 \mathrm{~nm}$ pores almost reaches the maximum limitation, and from Fig. 75, the thickness of adsorption layer in $10 \mathrm{~nm}$ pore is

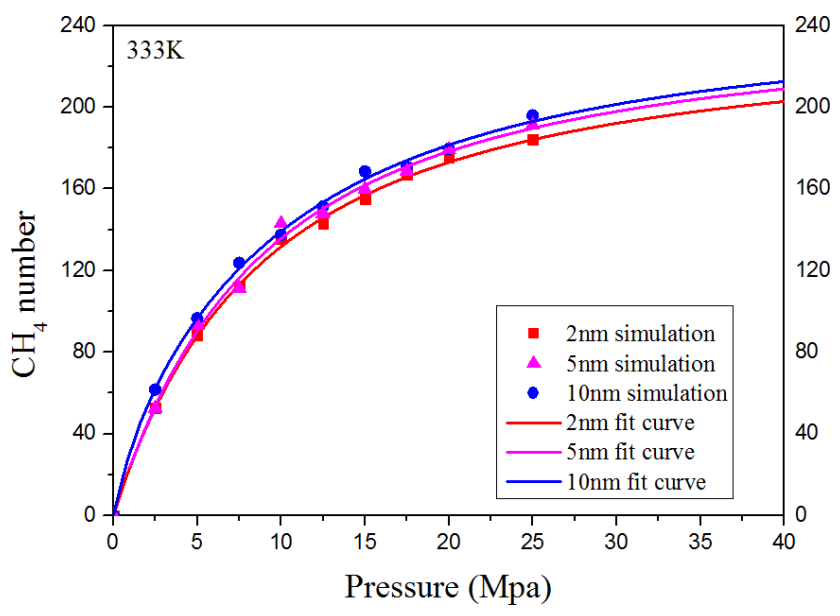

(a)

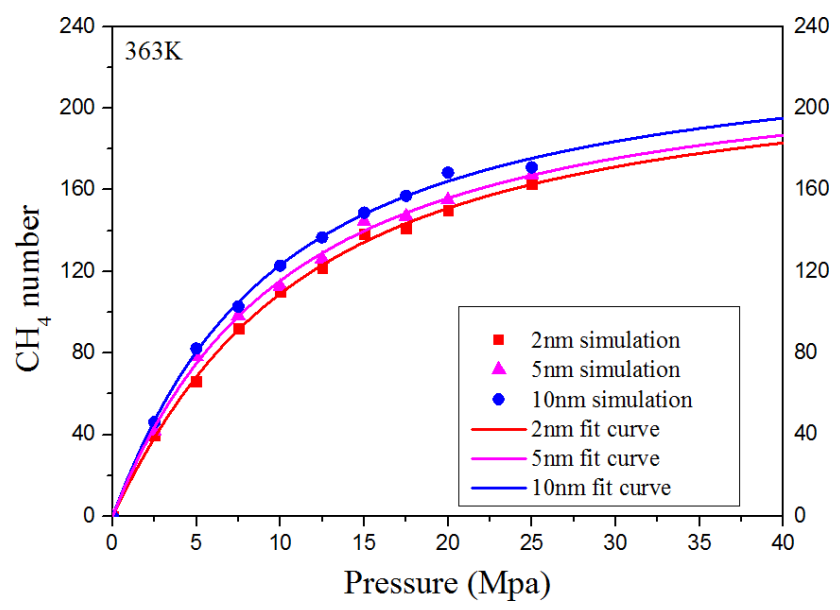

(b)

Fig. 8 Adsorption isothermal by simulation and BET fit. 
around $1 \mathrm{~nm}$, which is about three times larger than the methane diameter, so three layers of the adsorbed methane can be inferred, and compared with fitting data in Table 3, a fractal dimension of 2.31 can be inferred, pore wall with this roughness can have monolayer saturation adsorption number of 95.71 ; the thickness of adsorption layer in $2 \mathrm{~nm}$ pore and $5 \mathrm{~nm}$ pore is less than $1 \mathrm{~nm}$, we can infer a thickness near three times of methane diameter, and the fractal dimension of these two pores can be inferred as smaller than 2.63, and the monolayer saturation adsorption number is less than 107.23. Because of the same process of pore generation, different pore walls may have similar structure, the result of F-BET fitting shows the same trending, the pore wall fractal dimension is between 2.31 and 2.63, the monolayer saturation adsorption number is between 95.71 and 107.23, if more precise data can be simulated, we can calculate the fractal dimension of pore wall more accurately, and get the adsorption capacity of pore walls, and this is the job of future work.

\subsection{Impact of Pore Size on Adsorption Behavior}

The conclusion from Sec. 3.3 can be summarized: pore size is the determining factor of gas storage status, meanwhile, other factors include surface roughness and pore morphology. Since the methane is simulated by MD simulation, the results might be different from what come from lab tests. In the lab tests, the excess adsorption volume is the extra volume of absorbed gas to free gas in pores without surfaces of the same unit pore volume, the free gas density is calculated by theoretical method. The absolute adsorption volume is expressed as $n_{\mathrm{ab}}=n_{\mathrm{ex}}+n_{\text {free}}$. Due to the existence of pore surfaces, the interactions between pore surfaces and gas molecules increase average gas density and therefore, the free gas density in the kerogen pores is higher than that without the consideration of pore surfaces. Thus, the absolute adsorption volume calculated from lab tests is higher than the simulated one, which is more obvious for small pores. As shown in Fig. 9 the excess adsorption volume (horizontal line area) from lab tests on the right-hand side is higher than that from the simulation on the left-hand side; while the simulated absolute adsorption volume (vertical line area) shown on the left is smaller than that from the lab test on the right. This means, both excess and absolute adsorption

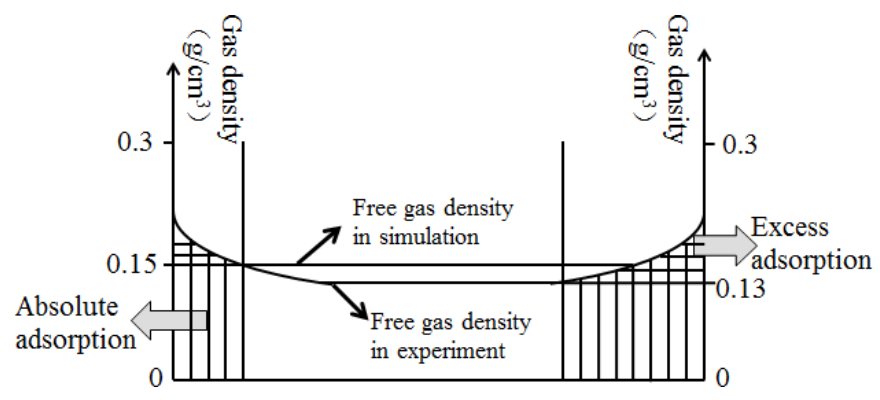

Fig. 9 Difference of adsorption between simulation and experiment (25 Mpa in $5 \mathrm{~nm}$ pore).

volumes are overestimated if pore surface impact is not considered. In reality, affected by the pore surfaces, free gas density in small pores is bigger than that in large pores. The adsorption behavior is only determined by the properties of pore surfaces, which certainly include pore geometry and matrix components.

\section{CONCLUSIONS}

The study in this paper begins with the construction of kerogen molecules, and then kerogen solid matrix and slit-like pores of different pore widths. With the combination of MD simulation and GCMC simulation, methane distribution in kerogen pores is acquired. Thereafter, multilayer adsorption behaviors on flat surfaces and rough surfaces are matched by the BET model and F-BET model, respectively. The conclusions from this study can be summarized as follows:

(1) Gas distribution in nanoscale pores is greatly affected by pore walls, the average gas density in the $2 \mathrm{~nm}$ pore is higher than that in $5 \mathrm{~nm}$ and $10 \mathrm{~nm}$ pores. Smaller pores can store more gas than bigger pores of the same pore volume. The absolute adsorption volume from the lab tests is higher than actual.

(2) Multilayer adsorption occurs in nanoscale pores. The number of adsorption layers in smaller pores is less than that in bigger pores and it increases with increase in pressure.

(3) Surface roughness is unavoidable during the construction of pores in kerogen, and it is reasonable to analyze the adsorption behavior by the multilayer fractal model. With increase of the fractal dimensions (more complex surface conditions), the same amount of adsorption gas occupies more layers and reduces free gas space. 


\section{ACKNOWLEDGMENTS}

The authors gratefully acknowledge the support given by the National Natural Science Foundation of China (Key Program) (Grant No. 51534006), the National Science Fund for Distinguished Young Scholars of China (Grant No. 51125019), the National Natural Science Foundation of China (Grant No. 5170427) and the Scientific Research Starting Project of SWPU (No. 2015QHZ003).

\section{REFERENCES}

1. R. H. Zhang et al., Simulation of a multistage fractured horizontal well with finite conductivity in composite shale gas reservoir through finite element method, Energy Fuels 30 (2016) 9036-9049.

2. Y. L. Zhao et al., "Triple porosity" modeling of transient well test and rate decline analysis for multifractured horizontal well in shale gas reservoirs, $J$. Petrol. Sci. Eng. 110 (2013) 253-262.

3. L. Chen et al., Quantitative characterization of micropore structure for organic-rich Lower Silurian shale in the Upper Yangtze Platform, South China: Implications for shale gas adsorption capacity, $A d v$. Geo-Energy Res. 1 (2017) 112-123.

4. Y. L. Zhao et al., Pressure response and production performance for multi-fractured horizontal wells with complex seepage mechanism in box-shaped shale gas reservoir, J. Nat. Gas Sci. Eng. 32 (2016) 66-80.

5. D. A. Wood and B. Hazra, Characterization of organic-rich shales for petroleum exploration \& exploitation: A review-Part 1: Bulk properties, multi-scale geometry and gas adsorption, J. Earth Sci. 28 (2017) 739-757.

6. Z. Zhang et al., Progress in enhancement of $\mathrm{CO} 2$ absorption by nanofluids: A mini review of mechanisms and current status, Renew. Energy 118 (2018) $527-535$.

7. C. Huang et al., Full-scale and multi-method combined characterization of micro/nano pores in organic shale, J. Nanosci. Nanotechnol. 17 (2017) 6634-6644.

8. L. Ji et al., Experimental investigation of main controls to methane adsorption in clay-rich rocks, Appl. Geochem. 27 (2012) 2533-2545.

9. D. J. Ross and R. M. Bustin, The importance of shale composition and pore structure upon gas storage potential of shale gas reservoirs, Mar. Pet. Geol. 26 (2009) 916-927.

10. G. R. Chalmers and R. M. Bustin, Lower cretaceous gas shales in northeastern British Columbia, Part I: Geological controls on methane sorption capacity, Bull. Can. Petrol. Geol. 56 (2008) 1-21.
11. S. Wang et al., The methane sorption capacity of Paleozoic shales from the Sichuan Basin, China, Mar. Pet. Geol. 44 (2013) 112-119.

12. W. Guo et al., Impact of temperature on the isothermal adsorption/desorption characteristics of shale gas, Petrol. Explor. Dev. 40 (2013) 481-485.

13. H. Liu and H. Wang, Adsorptivity and influential factors of marine shales in South China, Nat. Gas Ind. 32 (2012) 5-9.

14. L. Ji et al., Relationship between methane adsorption capacity of clay minerals and micropore volume, Nat. Gas Geosci. 25 (2014) 141-152.

15. K. Mosher et al., Molecular simulation of methane adsorption in micro- and meso-porous carbons with applications to coal and gas shale systems, Int. J. Coal Geol. 109 (2013) 36-44.

16. X. Q. Liu et al., Adsorption of $\mathrm{CH} 4$ on nitrogen- and boron-containing carbon models of coal predicted by density-functional theory, Appl. Surf. Sci. 285 (2013) 190-197.

17. L. Brochard et al., Adsorption-induced deformation of microporous materials: Coal swelling induced by $\mathrm{CO} 2-\mathrm{CH} 4$ competitive adsorption, Langmuir $\mathbf{2 8}$ (2012) 2659-2670.

18. J. Zhang et al., Combined Monte Carlo and molecular dynamics simulation of methane adsorption on dry and moist coal, Fuel 122 (2014) 186-197.

19. Y. Sawa et al., Pore-filling nature of $\mathrm{CH} 4$ adsorption behavior in kerogen nanopores: A molecular view based on full-atom kerogen models, in SPE Asia Pacific Unconventional Resources Conf. Exhibition (Society of Petroleum Engineers, 2015), pp. $1-13$.

20. J. Collell et al., Molecular simulation and modelisation of methane/ethane mixtures adsorption onto a microporous molecular model of kerogen under typical reservoir conditions, Microporous Mesoporous Mater. 197 (2014) 194-203.

21. M. Vandenbroucke, Kerogen: From types to models of chemical structure, Oil Gas Sci. Technol. 58 (2003) 243-269.

22. S. Plimpton, Fast parallel algorithms for short-range molecular dynamics, J. Comput. Phys. 117 (1995) $1-19$.

23. P. Dauberosguthorpe et al., Structure and energetics of ligand binding to proteins: Escherichia coli dihydrofolate reductase-trimethoprim, a drugreceptor system, Proteins 4 (1988) 31-47.

24. M. Gasparik et al., High-pressure methane sorption isotherms of black shales from the Netherlands, Energy Fuels 26 (2012) 4995-5004.

25. W. Humphrey, A. Dalke and K. Schulten, VMD: Visual molecular dynamics, J. Mol. Graph. 14 (1996) 33-38. 
26. W. A. Steele, The Interaction of Gases with Solid Surfaces (Pergamon Press, Oxford, 1974), pp. 348349.

27. C. Oostenbrink et al., A biomolecular force field based on the free enthalpy of hydration and solvation: The GROMOS force-field parameter sets 53A5 and 53A6, J. Comput. Chem. 25 (2004) 1656-1676.

28. J. I. Siepmann and D. Frenkel, Configurational bias Monte Carlo: A new sampling scheme for flexible chains, Mol. Phys. 75 (1992) 59-70.

29. D. Frenkel, G. Mooij and B. Smit, Novel scheme to study structural and thermal properties of continuously deformable molecules, J. Phys., Condens. Matter 4 (1992) 3053.

30. D. Frenkel and B. Smit, Understanding Molecular Simulation: From Algorithms to Applications (Academic Press, 2001), pp. 126-130.

31. S. Hao et al., Methane adsorption characteristics on coal surface above critical temperature through Dubinin-Astakhov model and Langmuir model, Colloids. Surf. A 444 (2014) 104-113.

32. P. Dutta, S. Harpalani and B. Prusty, Modeling of CO2 sorption on coal, Fuel 87 (2008) 2023-2036.

33. M. Richard, P. Bénard and R. Chahine, Gas adsorption process in activated carbon over a wide temperature range above the critical point. Part 1: Modified Dubinin-Astakhov model, Adsorption 15 (2009) 43-51.

34. A. M. Czerny, P. Bénard and R. Chahine, Adsorption of nitrogen on granular activated carbon: Experiment and modeling, Langmuir 21 (2005) 2871-2875.

35. J. Luo et al., Influence of structural parameters on methane adsorption over activated carbon: Evaluation by using D-A model, Fuel 123 (2014) 241-247.
36. Y. Gensterblum et al., High-pressure $\mathrm{CH} 4$ and $\mathrm{CO} 2$ sorption isotherms as a function of coal maturity and the influence of moisture, Int. J. Coal Geol. 118 (2013) 45-57.

37. B. Yu and J. Li, Some fractal characters of porous media, Fractals 9 (2001) 365-372.

38. O. Y. Dinariev, Gas and liquid transport in porous media with fractal geometry, Fluid Dyn. 27 (1992) 682-688.

39. J. C. Cai et al., Fractal analysis of surface roughness of particles in porous media, Chin. Phys. Lett. 27 (2010) 024705.

40. K. J. Falconer, Fractal geometry: Mathematical foundations and applications, Biometrics 46 (1990) 886.

41. J. R. Garrison, W. C. Pearn and D. U. Von Rosenberg, The fractal nature of geological data sets: Power law processes everywhere, in SPE Annual Technical Conf. Exhibition (Society of Petroleum Engineers, 1991), pp. 1-12.

42. D. C. Poon, M. McCormack and H. F. Thimm, The application of fractal geostatistics to oil and gas property evaluation and reserve estimates, J. Can. Petrol. Technol. 32 (1993) 24-27.

43. J. J. Fripiat, L. Gatineau and H. Van Damme, Multilayer physical adsorption on fractal surfaces, Langmuir 2 (1986) 562-567.

44. L. H. Zhang et al., Fractal pore structure model and multilayer fractal adsorption in shale, Fractals $\mathbf{2 2}$ (2014) 1-10.

45. P. E. T. Vajda and A. Felinger, Multilayer adsorption on fractal surfaces, J. Chromatogr. A 1324 (2014) 121-127.

46. D. D. Do, Adsorption analysis: Equilibria and Kinetics (Imperial College Press, London, 1998), p. 163. 